\title{
01.3
}

\section{Магнитный аналог вращения Квинке}

\author{
() А.И. Грачев \\ Физико-технический институт им. А.Ф. Иофффе РАН, Санкт-Петербург, Россия \\ E-mail: grach.shuv@mail.ioffe.ru
}

Поступило в Редакцию 23 сентября 2020 г.

В окончательной редакции 23 сентября 2020 г.

Принято к публикации 2 апреля 2021 г.

Обсуждается возможность наблюдения магнитного аналога вращения Квинке, т. е. спонтанного вращения в постоянном однородном магнитном поле (B) сферической частицы. Рассмотрено два варианта эксперимента: 1) слабомагнитная частица, погруженная в ферромагнитную жидкость; 2) диамагнитная сфера, левитирующая в вакууме. В первом случае спонтанное вращение возможно в полях $B<0.1 \mathrm{~T}$ для времен релаксации намагниченности частицы порядка $10^{-3} \mathrm{~s}$. Принципиальным условием ускоренного вращения диамагнитной сферы является ограничение ее вращательной степени свободы с единственно возможным направлением для оси вращения, ортогональной вектору В.

Ключевые слова: вращение Квинке, магнитная жидкость, диамагнитная сфера.

DOI: 10.21883/PJTF.2021.13.51116.18557

Вращение Квинке (ВК) - физическое явление, известное с конца XIX века $[1,2]$, которое заключается в спонтанном вращении частицы, находящейся в постоянном однородном электрическом поле. Направление оси вращения (вектора угловой скорости $\omega$ ) произвольно при условии ортогональности $\omega$ вектору электрического поля Е. Реализация ВК требует выполнения трех основных экспериментальных условий [2]: 1) частица должна быть помещена (диспергирована) в жидкую среду, электропроводность которой обеспечивает противоположное направление вектора дипольного момента частицы р относительно $\mathbf{E} ; 2)$ при повороте частицы поверхностный заряд, индуцирующий $\mathbf{p}$, должен перемещаться в том же направлении; 3) величина $\mathbf{E}$ должна превышать некоторое критическое значение $E_{c} \sim\left(\eta / \tau_{e l}\right)^{1 / 2}$, где $\eta-$ динамическая вязкость жидкости, а $\tau_{e l}$ - время релаксации поверхностного заряда. Два первых условия отвечают выполнению неравенства $\tau_{l}<\tau_{p}$, где $\tau_{l}$ и $\tau_{p}$ - время максвелловской релаксации для жидкости и частицы соответственно. Целью настоящей работы является демонстрация принципиальной возможности наблюдения аналога ВК - эффекта спонтанного вращения (сферической) частицы, помещенной в постоянное однородное магнитное поле (именуемого далее „магнитное вращение Квинке“ (МВК)) - на основе результатов качественного анализа двух довольно сильно различающихся физических моделей. Насколько нам известно, эффект МВК ранее в академической научной литературе не обсуждался, хотя явление вращения частиц во вращающемся магнитном поле и электровращение, являющееся его аналогом, известны достаточно давно, причем последнее, как, например, отмечается в [2], тесно связано с ВК.

Наш анализ естественно начать с рассмотрения модели так называемых магнитных дыр (МД), представляющих собой диамагнитную или парамагнитную микрочастицу, погруженную в (ферро)магнитную жидкость [3-5]. В магнитном поле магнитный момент МД $\mathbf{m}_{h}$ равен общему моменту ферромагнитных наночастиц, занимающих тот же объем, что и МД, причем $\mathbf{m}_{h}$ и вектор магнитного поля В антипараллельны друг другу. Для начала сравним крутящие моменты $\mathbf{T}_{e}=(\mathbf{p} \times \mathbf{E})$ и $\mathbf{T}_{m}=\left(\mathbf{m}_{h} \times \mathbf{B}\right)$, возникающие при небольшом повороте сферических частиц одинакового размера, отвечающие в случае электрического поля условию ВК, а в случае магнитной жидкости - области линейности зависимости $\mathbf{M}_{l}$ от В соответственно:

$$
\widehat{R}=T_{e} / T_{m}=3 \varepsilon_{0} \mu_{0} E^{2} / 2 \chi_{l} B^{2}
$$

где $\varepsilon_{0}$ и $\mu_{0}$ - диэлектрическая и магнитная проницаемости вакуума, а $\chi_{l}$ - магнитная восприимчивость магнитной жидкости. Используя данные [3] для магнитной жидкости $\left(B=10^{-2} \mathrm{~T}\right.$ и $\left.\chi_{l}=0.1\right)$ и предполагая, что $E \sim E_{c}=10^{6} \mathrm{~V} \cdot \mathrm{m}^{-1}$, получаем, что $\widehat{R} \approx 1.5$. Эффект ВК наблюдается в суспензиях с вязкостью $\eta \sim 10^{-2} \mathrm{~Pa} \cdot \mathrm{s}$ при значениях $\tau_{e l} \sim 10^{-3}$ s. C учетом того, что для магнитных жидкостей значение $\eta$ в лучшем случае $\sim 10^{-3} \mathrm{~Pa} \cdot \mathrm{s}$, реализация МВК (в указанной области магнитных полей) требует обеспечения времени релаксации намагниченности МД того же порядка, что и $\tau_{e l}$.

Релаксация $\mathbf{M}_{l}$, например, во вращающемся магнитном поле определяется двумя конкурирующими процессами: броуновским движением ферромагнитных наночастиц, вызывающим поворот частицы (и ее магнитного момента) как целого, и внутренним неелевским вращением намагниченности частицы с соответствующими характерными временами $\tau_{B}$ и $\tau_{N}$ [3]. Величина $\tau_{B}$ и $\tau_{N}$ зависит от размера наночастиц, и, согласно данным [6], время $\tau_{r e l}=\left(\tau_{B}^{-1}+\tau_{N}^{-1}\right)^{-1}$ достигает значений $\sim 10^{-3} \mathrm{~s}$ для частиц, радиус которых превышает 15-20 nm. Здесь, однако, следует учесть, что для механизма релаксации 

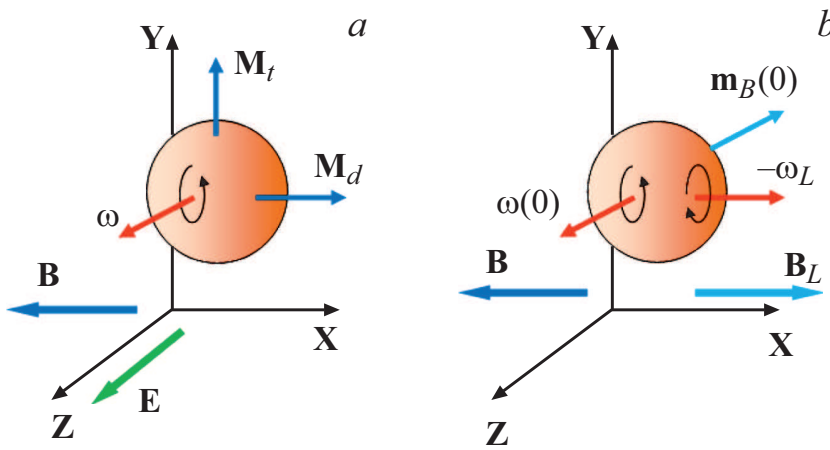

Схема вращения в постоянном однородном магнитном поле диамагнитной сферы, левитирующей в вакууме. $a-$ в условиях ограничения ее вращательной степени свободы (путем приложения постоянного электрического поля $\mathbf{E}$ ) с единственно возможным направлением оси вращения $(\| \mathbf{Z})$; $b$ - при отсутствии указанного ограничения. В начальный момент времени $t=0$ в результате случайной флуктуации сфера приобретает момент количества движения $\delta \mathbf{L} \| \mathbf{Z}$ и соответственно скорость вращения $\omega(0)=I^{-1} \delta \mathbf{L}(I-$ момент инерции сферы).

намагниченности МД существенное значение должна иметь адгезия наночастиц, причем их закреплению на поверхности МД и соответственно росту времени релаксации в значительной степени будет способствовать наличие каверн, трещин и других шероховатостей поверхности.

Таким образом, спонтанное вращение магнитных дыр в принципе реализуемо для ферромагнитных жидкостей в области полей $B<0.1 \mathrm{~T}$. Поскольку одной из основных целей исследования вращения Квинке являлось создание электростатического микромотора [2], рассмотренный эффект может в свою очередь стимулировать разработку его магнитостатического аналога.

Другой вариант МВК (см. рисунок, $a)$ - диамагнитная непроводящая сфера, левитирующая в среде со сколь угодно низкой вязкостью (в вакууме), - специфичен именно для магнитного взаимодействия. Нетрудно увидеть, что в этом случае „автоматически“ выполнены два первых условия для наблюдения МВК. Действительно, магнитный момент частицы $\mathbf{m}_{d}=V \mathbf{M}_{d}=\chi_{d} \mu_{0}^{-1} \mathbf{B} V$ (где $\mathbf{M}_{d}$ - равновесная намагниченность, $\chi_{d}$ - диамагнитная восприимчивость, $V$ - объем сферы), индуцируемый магнитным полем В, антипараллелен последнему. При (случайном) повороте частицы относительно направления $\mathbf{B}$ вектор $\mathbf{m}_{d}$ меняется не мгновенно, а с характерным временем релаксации намагниченности $\tau_{d}$. Поэтому на протяжении определенного временно́го интервала сохраняется компонента $\mathbf{m}_{d}$, ортогональная $\mathbf{B}$, и существует момент сил, работающий на дальнейшее увеличение отклонения. Наконец, разумные значения критического поля $B_{c}=\left(\mu_{0} \eta / \chi_{d} \tau_{d}\right)^{1 / 2}$ могут быть обеспечены для достаточно коротких $\tau_{d}$ при конечной величине $\eta$. Например, при $B_{c} \sim 0.1 \mathrm{~T}, \chi_{d} \sim 10^{-5}$ и $\tau_{d} \sim 10^{-9} \mathrm{~s}$ величина $\eta \sim 10^{-11} \mathrm{~Pa} \cdot \mathrm{s}$, что отвечает вязкости воздуха при давлении $\sim 0.1$ Ра. Таким образом, все необходимые условия для наблюдения МВК выполнены. Однако следует вспомнить о различии динамики частиц, например, с постоянным электрическим или магнитным моментом, неколлинеарным Е и В соответственно. В первом случае частица поворачивается вокруг оси, ортогональной $\mathbf{E}$, а во втором совершает прецессию вокруг В с угловой (ларморовской) частотой $\omega_{L}=-\gamma \mathbf{B}$, где $\gamma-$ гиромагнитное отношение. Поэтому необходимо ограничить вращательную степень свободы диамагнитной сферы лишь одной осью вращения, ортогональной $\mathbf{H}$ (ось $\mathbf{Z}$ на рисунке, $a$ ) (отметим, что в магнитной жидкости ларморовской прецессии МД препятствует вязкое сопротивление среды [3]). Бесконтактно ограничить вращение сферы можно, приложив постоянное электрическое поле $\mathbf{E} \| \mathbf{Z}$ (см. рисунок, $a$ ), а необходимое соотношение между величиной последнего и полем В грубо можно оценить, используя выражение $(1)$, откуда при $\chi_{d} \sim 10^{-5}$ получаем $E\left[\mathrm{~V} \cdot \mathrm{m}^{-1}\right]>10^{6} B[\mathrm{~T}]$.

Из анализа вращения частиц в постоянном магнитном поле, например, для случая вращения магнитной жидкости как целого [6] следует вывод о появлении компоненты намагниченности $\mathbf{M}_{t} \perp \mathbf{B}$, которая при $\omega \tau_{r e l} \ll 1$ равна $M_{t}(t)=\omega(t) \tau_{r e l} M_{0}$, где $M_{0}$ - равновесная намагниченность. Допустим теперь, что в результате соударения с некой частицей (в момент времени $t=0)$ покоившаяся до того сфера приобретает момент количества движения $\delta \mathbf{L} \| \mathbf{Z}$. Это вызовет ее вращение с начальной скоростью $\omega(0)=\delta L / I$ ( $I-$ момент инерции сферы), и в отсутствие вязкого сопротивления среды и условия $\omega \tau_{d} \ll 1$ дальнейшее движение частицы описывается уравнением

$$
I d \omega / d t=V\left(\mathbf{M}_{t} \times \mathbf{B}\right)=\mu_{0}^{-1} \chi_{d} V \omega \tau_{d} B^{2} .
$$

Видно, что на начальном этапе скорость вращения растет экспоненциально: $\omega(t)=\omega(0) \exp \left(t / \tau_{\text {rot }}\right)$ с $\tau_{r o t}=\mu_{0} 2 \rho R^{2} / 5 \chi_{d} \tau_{d} B^{2}$, где $\rho$ и $R$ - плотность и радиус сферы. Для величины $\tau_{\text {rot }}$ частицы с $R \sim 0.1 \mu \mathrm{m}$ и значениях остальных величин $\chi_{d} \sim 10^{-5}$, $B \sim 0.1 \mathrm{~T}, \rho \sim 2.5 \cdot 10^{3} \mathrm{~kg} \cdot \mathrm{m}^{-3}$ и $\tau_{d} \sim 10^{-9} \mathrm{~s}$ получаем $\tau_{\text {rot }} \sim 10^{-1} \mathrm{~s}$.

Общее описание динамики сферы и изменения вектора намагниченности $\mathbf{M}(t)$ требует решения системы уравнений, аналогичной случаю ВК [7]:

$$
\begin{gathered}
I d \omega / d t=V(\mathbf{M}(t) \times \mathbf{B})-6 \eta V \omega, \\
d \mathbf{M} / d t=(\omega \times \mathbf{M})-\left(\mathbf{M}-\mathbf{M}_{d}\right) / \tau_{d} .
\end{gathered}
$$

Предположим теперь, что флуктуация $\delta \mathbf{L}$ произошла в отсутствие поля $\mathbf{E}$ (см. рисунок, $b$ ). Теперь $\mathbf{M}_{t}$ совместно с моментом $\mathbf{m}_{B}=\chi_{d}\left(\mu_{0} \gamma\right)^{-1} \boldsymbol{\omega}(0)$ (на рисунке, $a$ не показан) вызовет начало ларморовской прецессии. Далее исходя из общих соображений эволюция вращения и намагниченности частицы при условии $\eta=0$ будет выглядеть следующим образом. Установившаяся прецессия приведет к появлению поля $\mathbf{B}_{L}=-\gamma^{-1} \omega_{L}$, равного по 
абсолютной величине В, поэтому продольная составляющая намагниченности частицы исчезнет. В результате сфера одновременно с прецессией будет испытывать вращение вокруг двух других осей таким образом, что $\left|\mathbf{m}_{B}(t)\right| \sim|\omega(t)|=\left|\omega_{Z}(t)+\omega_{Y}(t)\right|=\operatorname{const}(t)$. В случае конечного значения $\eta$ вращение частицы рано или поздно должно полностью прекратиться. Таким образом, в отсутствие указанного ограничения вращательной степени свободы флуктуации момента количества движения сферы не только не могут вызвать ее последующего ускоренного вращения (т. е. эффект МВК), но и должны со временем затухать.

Практическая реализация экспериментальной схемы, показанной на рисунке, $a$, может представлять интерес для изучения таких фундаментальных явлений, как квантовое трение в вакууме, трение Казимира и пр. [8]. На данный момент максимальная скорость $\omega_{\max } \sim 10^{9} \mathrm{~s}^{-1}$ частиц микронного размера достигнута в экспериментах с использованием оптических пинцетов [9], способных одновременно осуществлять вращение частиц и их левитацию. Использование диамагнитных частиц, помещенных в неоднородное магнитное поля, позволяет реализовать механизм их пассивной левитации, причем, приложив неоднородное электрическое поле, в принципе можно усилить эффект левитации, если его максимум пространственно совмещен с минимумом магнитного поля. Все это позволяет надеяться на то, что в случае развития данной методики она сможет составить конкуренцию оптическим пинцетам, хотя интересным может оказаться также и их совместное использование.

\section{Конфликт интересов}

Автор заявляет, что у него нет конфликта интересов.

\section{Список литературы}

[1] G. Quincke, Ann. Phys. Chem. Band., 59, 417 (1896).

[2] T.B. Jones, Electromechanics of particles, 1st ed. (Cambridge University Press, N.Y., 1995).

[3] М.И. Шлиомис, УФН, 112 (3), 427 (1974).

[4] M.C. Miguel, J.M. Rubi, Physica A, 231 (1-3), 288 (1996). DOI: 10.1016/0378-4371(96)00077-5

[5] B.E. Kashevsky, Phys. Fluids, 9(6), 1811 (1997). DOI: $10.1063 / 1.869296$

[6] J.P. Embs, B. Huke, A. Leschhorn, M. Lücke, Z. Phys. Chem., 222 (2-3), 527 (2008). DOI: 10.1524/zpch.2008.222.2-3.527

[7] N. Pannacci, L. Lobry, E. Lemaire, Phys. Rev. Lett., 99 (9), 094503 (2007). DOI: 10.1103/PhysRevLett.99.094503

[8] Г.В. Дедков, А.А. Кясов, УФН, 187 (6), 599 (2017). DOI: $10.3367 /$ UFNr.2016.12.038006R

[9] R. Reimann, M. Doderer, E. Hebestreit, R. Diehl, M. Frimmer, D. Windey, F. Tebbenjohanns, L. Novotny, Phys Rev. Lett., 121 (3), 033602 (2018). DOI: 10.1103/PhysRevLett.121.033602 